PROCEEDINGS OF THE AMERICAN MATHEMATICAL SOCIETY

Volume 00, Number 0, Xxxx 19XX

\title{
REPRESENTING THE AUTOMORPHISM GROUP OF AN ALMOST CRYSTALLOGRAPHIC GROUP
}

\author{
PAUL IGODT AND WIM MALFAIT \\ (Communicated by Ron Solomon)
}

\begin{abstract}
Let $E$ be an almost crystallographic (AC-) group, corresponding to the simply connected, connected, nilpotent Lie group $L$ and with holonomy group $F$. If $L^{F}=\{1\}$, there is a faithful representation $\operatorname{Aut}(E) \hookrightarrow \operatorname{Aff}(L)$. In case $E$ is crystallographic, this condition $L^{F}=\{1\}$ is known to be equivalent to $Z(E)=1$ or $b_{1}(E)=0$. We will show (Example 2.2) that, for AC-groups $E$, this is no longer valid and should be adapted. A generalised equivalent algebraic (and easier to verify) condition is presented (Theorem 2.3). Corresponding to an AC-group $E$ and by factoring out subsequent centers we construct a series of AC-groups, which becomes constant after a finite number of terms. Under suitable conditions, this opens a way to represent $\operatorname{Aut}(E)$ faithfully in $\operatorname{Gl}(k, \mathbb{Z}) \times \operatorname{Aff}\left(L_{1}\right)$ (Theorem 4.1). We show how this can be used to calculate $\operatorname{Out}(E)$. This is of importance, especially, when $E$ is almost Bieberbach and, hence, $\operatorname{Out}(E)$ is known to have an interesting geometric meaning.
\end{abstract}

\section{Preliminaries}

Let us fix some notation here. If $G$ is a group and $x, y \in G$, we will use the following conventions: $[x, y]=x^{-1} y^{-1} x y, x^{y}=y^{-1} x y$. The following commutator identities are rather well known, and will be used later on.

(1)

$$
\forall x, y \in G: \forall m \in \mathbb{N}_{0}:\left[x^{m}, y\right]=\prod_{j=1}^{m}[x, y]^{x^{m-j}} \text { and } \quad\left[x, y^{m}\right]=\prod_{j=0}^{m-1}[x, y]^{y^{j}} .
$$

We will write $\mu(x)$ for the inner automorphism of $G$ determined by $x$; i.e. $\mu(x)(y)=$ $x y x^{-1}$.

Recall that the lower central series of $G$ is defined inductively by $\gamma_{1}(G)=G$ and $\gamma_{n+1}(G)=\left[\gamma_{n}(G), G\right]\left(n \in \mathbb{N}_{0}\right)$. G is said to be $c$-step nilpotent (or nilpotent of class $c$ ) if and only if $\gamma_{c}(G) \neq\{1\}$ and $\gamma_{c+1}(G)=\{1\}$. It then follows that $\gamma_{c}(G) \subseteq Z(G)$. Furthermore, its upper central series is defined inductively by $Z_{0}(G)=\{1\}$ and $Z_{n+1}(G) / Z_{n}(G)=Z\left(G / Z_{n}(G)\right)(n \in \mathbb{N})$.

Received by the editors May 5, 1994.

1991 Mathematics Subject Classification. Primary 20H15, 20F34, 20 F28.

Key words and phrases. Almost crystallographic group, automorphism group, outer automorphism group.

The second author is Research Assistant of the National Fund For Scientific Research (Belgium). 
We recall also briefly the concept of the isolator (sometimes called the root set) of a subgroup in a group.

Definition 1.1 ([Pas77], [Seg83]). Let $G$ be a group and $H$ a subgroup of $G$. The isolator of $H$ in $G$ is defined by

$$
\sqrt[G]{H}=\left\{g \in G \| g^{k} \in H \text { for some } k \geq 1\right\}
$$

It is well known that, for every group $G, \sqrt[G]{\gamma_{k}(G)}$ is a characteristic subgroup of $G$ and

$$
\left[\sqrt[G]{\gamma_{k}(G)}, \sqrt[G]{\gamma_{l}(G)}\right] \subseteq \sqrt[G]{\gamma_{k+l}(G)}
$$

The first Betti number of a finitely generated group $G$, written as $b_{1}(G)$, is defined as the torsion-free rank of its abelianised group $G / \gamma_{2}(G)$. Now, it is clear that, if $G$ is a finitely generated group and $\mathbb{Z}^{k}$ is a trivial $G$-module, then

$$
Z^{1}\left(G, \mathbb{Z}^{k}\right)=H^{1}\left(G, \mathbb{Z}^{k}\right) \cong \mathbb{Z}^{k b_{1}(G)} .
$$

Consequently,

Lemma 1.2. Let $G$ be a finitely generated group with torsion-free center $Z(G)$. If $b_{1}(G / Z(G)=0$, then $Z(G / Z(G)=\{1\}$.

Proof. Take $x Z(G) \in Z(G / Z(G), x \in G$. The inner automorphism $\mu(x)$ induces the identity on $Z(G)$ and on $G / Z(G)$. The subgroup of all such automorphisms of $G$ is isomorphic to $Z^{1}(G / Z(G), Z(G))$ (e.g. see [IM94]). Since $Z(G)$ is a torsion-free, trivial $G / Z(G)$-module and $b_{1}\left(G / Z(G)=0\right.$, we conclude that $Z^{1}(G / Z(G), Z(G))$ is trivial (use (2)) or $x \in Z(G)$.

\section{Automorphisms of Almost CRYSTAllographic groups I}

Let $L$ be a connected, simply connected, nilpotent Lie group. We write $\operatorname{Aff}(L)$ for the semi-direct product $L \rtimes \operatorname{Aut}(L) . \operatorname{Aff}(L)$ is called the group of affine diffeomorphisms of $L$ and acts in a natural way on $L$; for $x, y \in L$ and $\alpha \in \operatorname{Aut}(L)$, ${ }^{(x, \alpha)} y=x \alpha(y)$. Let $C$ be a compact subgroup of $\operatorname{Aut}(L)$. A uniform, discrete subgroup $E$ of $L \rtimes C \subset \operatorname{Aff}(L)$ is called an almost crystallographic (AC-) group (of $L)$. It is well known that $N=E \cap L$ ([Aus60]) is a torsion-free , finitely generated, nilpotent normal subgroup of finite index in $E$, which is maximal nilpotent in $E$. The finite group $F=E / E \cap L$, which is sometimes called the holonomy group, acts faithfully on $L$. The Hirsch length (rank) of $N$ is often referred to as the dimension of $E$.

As an abstract group, a group $E$ is $\mathrm{AC}$ if and only if it contains a torsion-free, finitely generated, nilpotent normal subgroup $N$ of finite index, which is maximal nilpotent in $E$. In this case, $N$ equals the Fitting subgroup Fitt $(E)$ of $E$, which is defined as the subgroup generated by all nilpotent normal subgroups of $E$ (see [Seg83]). In this case, the Lie group $L$ is the Mal'cev completion of $N$ ([Mal51]). Consequently, it is clear at once that isomorphic $\mathrm{AC}$-groups correspond to the same Lie group $L$.

A torsion-free AC-group is called an almost Bieberbach (AB-) group. AC-(resp. AB-) groups have been studied intensively as generalisations of classical crystallographic (resp. Bieberbach) groups (i.e. the situation with $L=\mathbb{R}^{k}$ ). In this perspective, the following theorem, which is a generalisation of the classical second Bieberbach theorem, can be found in [LR85]. 
Theorem 2.1. If $f: E \rightarrow E^{\prime}$ is an isomorphism of two AC-groups (of $L$ ), then $f$ can be realised as conjugation in $\operatorname{Aff}(L)$.

Let $L^{F}$ be the subset of $L$ consisting of points fixed under the action of $F$. One easily verifies that

$$
C_{\mathrm{Aff}(L)}(E)=\left\{\left(x, \mu\left(x^{-1}\right)\right) \in \operatorname{Aff}(L) \| x \in L^{F}\right\} \cong L^{F} .
$$

Consequently, if $L^{F}=\{1\}$, an isomorphism of two AC-groups (of $L$ ) can be realised in a unique way as an affine conjugation. Applied to a given AC-group $E$, one obtains a representation $\operatorname{Aut}(E) \hookrightarrow \operatorname{Aff}(L)$.

Unfortunately, in the usual algebraic setting, verifying if $L^{F}=\{1\}$ is often very hard, as one does not know the action of $F$ on $L$ explicitly. In the crystallographic case, however, a simple algebraic equivalent condition is known. To see this, note that $L^{F}$ contains the abelian, normal subgroup $Z(L)^{F}$. Also, $Z(E)$ is a uniform lattice of $Z(L)^{F}$. Therefore, $E$ is centerless if and only if $Z(L)^{F}=\{1\}$. Consequently, if $E$ is a centerless, $k$-dimensional, crystallographic group (in which case $L=Z(L)$ ) (or equivalently, if $E$ is crystallographic with first Betti number zero ([HS86])), each isomorphism between $E$ and another crystallographic group $E^{\prime}$ can be realised as conjugation by a unique element of $\operatorname{Aff}\left(\mathbb{R}^{k}\right)$. So, if $E$ is crystallographic with $b_{1}(E)=0$, there is a well-defined representation $\operatorname{Aut}(E) \hookrightarrow \operatorname{Aff}\left(\mathbb{R}^{k}\right)$.

As a first observation, we show with an example (more general for centerless AC-groups ( $L$ non-abelian $)$ ) that a similar faithful representation does not hold anymore; i.e. being centerless is not any more sufficient to imply that an automorphism of $E$ is realised as conjugation by a unique element in $\operatorname{Aff}(L)$. Remark that this does not imply that a representation $\operatorname{Aut}(E) \rightarrow \operatorname{Aff}(L)$ does not exist.

Example 2.2. Consider the Heisenberg group

$$
H=\left\{\left(\begin{array}{ccc}
1 & y & z \\
0 & 1 & x \\
0 & 0 & 1
\end{array}\right) \mid x, y, z \in \mathbb{R}\right\}
$$

which is connected, simply connected and nilpotent of class 2 . In $H$ consider

$$
A=\left(\begin{array}{lll}
1 & 0 & 0 \\
0 & 1 & 1 \\
0 & 0 & 1
\end{array}\right), B=\left(\begin{array}{lll}
1 & 1 & 0 \\
0 & 1 & 0 \\
0 & 0 & 1
\end{array}\right), C=\left(\begin{array}{lll}
1 & 0 & 1 \\
0 & 1 & 0 \\
0 & 0 & 1
\end{array}\right)
$$

In a connected, simply connected nilpotent Lie group $G$, it makes sense to speak about $g^{x}=\exp (x \log g)$, where $g \in G$ and $x \in \mathbb{R}$. So, we can say that $H=$ $\left\{A^{x} B^{y} C^{z} \| x, y, z \in \mathbb{R}\right\}$. Observe that, in $H$, the relations $[B, A]=C,[C, A]=1$ and $[C, B]=1$ hold. Also, remark that $Z(H)=\left\{C^{z} \| z \in \mathbb{R}\right\} \cong \mathbb{R}$.

Take the uniform lattice $N$ in $H$ generated by $a=A, b=B$ and $c=\sqrt{C}$. As a presentation for $N$, we have

$$
N:\left\langle a, b, c \|[b, a]=c^{2},[c, a]=[c, b]=1\right\rangle .
$$

Take $F \cong \mathbb{Z}_{2}$, given as $\{1, \alpha\}$, and let $F$ act on $N$ via the homomorphism $\varphi: F \rightarrow$ $\operatorname{Aut}(N)$ given by

$$
\varphi(\alpha): N \rightarrow N: a \mapsto a, b \mapsto b^{-1}, c \mapsto c^{-1} .
$$

This can be lifted uniquely to an action $\widetilde{\varphi}: F \rightarrow \operatorname{Aut}(H)$; then $\alpha$ sends $A \mapsto A$, $B \mapsto B^{-1}$ and $C \mapsto C^{-1}$. Clearly, $H^{F}=\left\{A^{x} \| x \in \mathbb{R}\right\} \cong \mathbb{R}$ while $Z(H)^{F}$ is trivial. 
Let $1 \rightarrow N \rightarrow E \cong N \rtimes F \rightarrow F \rightarrow 1$ be the semi-direct product determined by $\varphi$. E.g. a presentation of $E$ could be

$$
\begin{aligned}
E:\left\langle a, b, c, \alpha \|[b, a]=c^{2},[c, a]=[c, b]\right. & =1, \\
\alpha a & \left.=a \alpha, \alpha b=b^{-1} \alpha, \alpha c=c^{-1} \alpha, \alpha^{2}=1\right\rangle .
\end{aligned}
$$

It is not hard to see that $E$ is a 3-dimensional, centerless AC-group having $N$ as the 2-step nilpotent maximal nilpotent subgroup. An embedding $\imath$ of $E$ into $\operatorname{Aff}(H)=H \rtimes \operatorname{Aut}(H)$ is given by

$$
\imath: E \hookrightarrow \operatorname{Aff}(H): a \mapsto(A, 1), b \mapsto(B, 1), c \mapsto(\sqrt{C}, 1), \alpha \mapsto(1, \widetilde{\varphi}(\alpha)) .
$$

The following automorphism of $E$

$$
\sigma: E \rightarrow E: a \mapsto a c, b \mapsto b, c \mapsto c, \alpha \mapsto b \alpha
$$

can be realised as conjugation by the affinities $\left(h_{\sigma}, \alpha_{\sigma}\right) \in H \rtimes \operatorname{Aut}(H)$, where $h_{\sigma}=$ $A^{x} B^{\frac{1}{2}} C^{\frac{x}{2}}$ and $\alpha_{\sigma}: H \rightarrow H$ sends $A \mapsto A, B \mapsto B C^{x}$ and $C \mapsto C$, for each $x \in \mathbb{R}$.

Our next aim is to present a necessary and sufficient condition for the situation $L^{F}=\{1\}$, given an AC-group $E$ of $L$ with holonomy $F$.

From now on, we assume that $1 \rightarrow N \rightarrow E \rightarrow F \rightarrow 1$ is a short exact sequence of groups where $N$ is finitely generated, torsion-free, $c$-step nilpotent of finite index in $E$ and maximal nilpotent in $E$. These extensions have been called "essential" ([Lee88]) or "strict normal" ([GS92]).

Define the following quotients:

$$
\tau_{i}(N)=N / \sqrt[N]{\gamma_{i+1}(N)} \text { and } \tau_{i}(E)=E / \sqrt[N]{\gamma_{i+1}(N)}, 1 \leq i \leq c
$$

It is known that $1 \rightarrow \tau_{c-1}(N) \rightarrow \tau_{c-1}(E) \rightarrow F \rightarrow 1$ is again essential ([DIM93]) and that $\tau_{c-1}(N)$ is nilpotent of class $c-1$. Obviously, $\tau_{j}\left(\tau_{c-1}(E)\right)\left(\tau_{j}\left(\tau_{c-1}(N)\right)\right)$ is isomorphic to $\left.\tau_{j}(E)\left(\tau_{j}(N)\right)\right)(1 \leq j \leq c-1)$, and hence, by induction, all extensions $1 \rightarrow \tau_{i}(N) \rightarrow \tau_{i}(E) \rightarrow F \rightarrow 1(1 \leq i \leq c)$ are essential.

Theorem 2.3. Let $E$ be an AC-group given by an essential extension $1 \rightarrow N \rightarrow$ $E \rightarrow F \rightarrow 1$ where $N$ is c-step nilpotent, and assume $L$ is the Mal'cev completion of $N$. Then, $L^{F}=\{1\}$ if and only if all AC-groups $\tau_{i}(E)(1 \leq i \leq c)$ are centerless.

Proof. In the abelian case $(c=1)$, we already observed that $E$ is centerless if and only if $L^{F}=\{1\}$.

We proceed by induction on $c$. As mentioned above, $\tau_{c-1}(E)$ is an AC-group with Fitting subgroup $\tau_{c-1}(N)$. This is a $(c-1)$-step nilpotent group with Mal'cev completion $L / \gamma_{c}(L)$. Remark that all AC-groups $\tau_{j}\left(\tau_{c-1}(E)\right) \cong \tau_{j}(E)(1 \leq j \leq$ $c-1)$ are centerless. Hence, by induction, $\left(L / \gamma_{c}(L)\right)^{F}=\{1\}$ or $L^{F}=\gamma_{c}(L)^{F}$. Now, because $L$ is $c$-step nilpotent $\left(\gamma_{c}(L) \subset Z(L)\right)$ and $E$ is centerless $\left(Z(L)^{F}=\{1\}\right)$, we can conclude that $L^{F}$ is trivial.

To prove the converse, first observe that it will be sufficient to show that, if $L^{F}=$ $\{1\}$, then $\left(L / \gamma_{c}(L)\right)^{F}=\{1\}$. This will imply, by induction, that all $\tau_{j}\left(\tau_{c-1}(E)\right) \cong$ $\tau_{j}(E)(1 \leq j \leq c-1)$ are centerless. Added to the fact that, if $L^{F}$ is trivial, then also $\tau_{c}(E)=E$ is centerless, this then finishes the claim.

Let $k$ be the order of $F$ and assume $\ell \gamma_{c}(L)$ is a fixed point for the action of $F$ on $L / \gamma_{c}(L)$. Define a (normalised) 1-cochain $\lambda: F \rightarrow \gamma_{c}(L) \subset Z(L)$ as follows: for $x \in F,{ }^{x} \ell=\ell \lambda(x)$. It is easily verified that $\lambda: F \rightarrow Z(L)$ is a 1-cocycle. 
As $L$ and $Z(L)$ are both divisible with unique roots, one can consider the element $\ell_{0}=\prod_{y \in F} \lambda(y)^{\frac{1}{k}} \in Z(L)$. Now, verify that, for $x \in F$,

$$
{ }^{x} \ell_{0}=\prod_{y \in F}{ }^{x} \lambda(y)^{\frac{1}{k}}=\prod_{y \in F}\left(\lambda(x y) \lambda(x)^{-1}\right)^{\frac{1}{k}}=\prod_{y \in F} \lambda(x y)^{\frac{1}{k}} \prod_{y \in F} \lambda(x)^{-\frac{1}{k}}=\ell_{0} \lambda(x)^{-1} .
$$

Consequently $\lambda: F \rightarrow Z(L)$ is a 1-coboundary. Hence, for all $x \in F$,

$$
{ }^{x}\left(\ell \ell_{0}\right)=\ell \lambda(x){ }^{x} \ell_{0}=\ell \ell_{0}
$$

and there is a fixed point for the action of $F$ on $L$. This contradicts the assumption. We conclude that $\left(L / \gamma_{c}(L)\right)^{F}=\{1\}$.

Corollary 2.4. Let $E$ be an AC-group given by an essential extension $1 \rightarrow N \rightarrow$ $E \rightarrow F \rightarrow 1$ as above. If all $A C$-groups $\tau_{i}(E)(1 \leq i \leq c)$ are centerless, then there is a well-defined faithful representation $\operatorname{Aut}(E) \hookrightarrow \operatorname{Aff}(L)$.

\section{The AC-SERIES OF AN ALmost CRYSTALlographiC GROUP}

It is well known (e.g. [DIM93, Corollary 5.5]) that, if $1 \rightarrow N \rightarrow E \rightarrow F \rightarrow 1$ is an essential extension, the corresponding abstract kernel $\psi: F \rightarrow \operatorname{Out}(N)$ is injective. For such an extension it then follows that $C_{E}(N)=Z(N)$ and, hence, that $Z(E)$ is a normal subgroup of $N$. This allows us to state the following

Proposition 3.1. If $1 \rightarrow N \rightarrow E \rightarrow F \rightarrow 1$ is an essential extension, then $1 \rightarrow$ $N / Z(E) \rightarrow E / Z(E) \rightarrow F \rightarrow 1$ is essential too.

Proof. Let us first show that $Z(N) / Z(E)$ is torsion-free. Assume $x \in Z(N)$ and $x^{m} \in Z(E)$, for $m \geq 2$. Then, for each $y \in E$,

$$
1=\left[x^{m}, y\right] \stackrel{(1)}{=} \prod_{j=1}^{m}[x, y]^{x^{m-j}}=[x, y]^{m}
$$

and, as $N$ is torsion-free, $x \in Z(E)$.

Now, $1 \rightarrow Z(N) / Z(E) \rightarrow N / Z(E) \rightarrow N / Z(N) \rightarrow 1$ shows that $N / Z(E)$ is a central extension of a torsion-free, abelian group by a torsion-free nilpotent group. Therefore $N / Z(E)$ itself is torsion-free and, of course, nilpotent of class $\leq c$ (if $N$ is of class $\leq c)$.

$N / Z(E)$ is quite easily seen to be maximal nilpotent in $E / Z(E)$. Indeed, suppose $N^{\prime}$ is a subgroup of $E$ containing $N$ such that $N^{\prime} / Z(E)$ is nilpotent. Then $1 \rightarrow$ $Z(E) \rightarrow N^{\prime} \rightarrow N^{\prime} / Z(E) \rightarrow 1$ is a central extension by a nilpotent group and consequently $N^{\prime}$ is nilpotent. This contradicts the maximal nilpotency of $N$.

This allows the introduction of the following

Definition 3.2. For an AC-group $E$ given by an essential extension $1 \rightarrow N \rightarrow$ $E \rightarrow F \rightarrow 1$, we define the associated AC-series $\left(E_{i}\right)_{i \in \mathbb{N}}$ inductively as follows:

$$
E_{0}=E, E_{i+1}=E_{i} / Z\left(E_{i}\right) \cong \operatorname{Inn}\left(E_{i}\right) .
$$

Remark 3.3. By defining, in a similar way, $N_{0}=N, N_{i+1}=N_{i} / Z\left(E_{i}\right)$, it is clear that each extension $1 \rightarrow N_{i} \rightarrow E_{i} \rightarrow F \rightarrow 1$ is essential and hence the groups $E_{i}$ are AC-groups. This motivates the term AC-series. 
As could be expected from the definition of the AC-series, there is an interesting and nice relation of this concept and the upper central series terms of $E$ and $N$. We summarise as follows:

Lemma 3.4. Assume $1 \rightarrow N \rightarrow E \rightarrow F \rightarrow 1$ is an essential extension, and $\left(E_{i}\right)_{i}$ is the associated $A C$-series of $E$.

(1) $\forall i: E_{i} \cong E / Z_{i}(E)$ and $N_{i} \cong N / Z_{i}(E)$.

(2) $\forall i: Z_{i}(E) \subset Z_{i}(N)$.

(3) $\forall i:\left(Z_{i+1}(E) \backslash Z_{i}(E)\right) \subset\left(Z_{i+1}(N) \backslash Z_{i}(N)\right)$.

(4) If $c$ is the nilpotency class of $N$, then, after at most $c$ steps, the $A C$-series $\left(E_{i}\right)_{i}$ becomes constant.

Proof. (1) The first part is obvious from the definition of $E_{i}$. Since $E_{i} / N_{i} \cong E / N \cong$ $F$, we conclude that $N_{i} \cong N / Z_{i}(E)$.

(2) Use induction on $i$. If $x \in Z_{i+1}(E)$, then $x \in N$ and $x Z_{i}(E) \in Z\left(E / Z_{i}(E) \cong\right.$ $\left.E_{i}\right)$. Hence, $[x, E] \subset Z_{i}(E) \subset Z_{i}(N)$ and consequently also $[x, N] \subset Z_{i}(N)$, which means that $x \in Z_{i+1}(N)$.

(3) Again we proceed by induction on $i$. For $x \in Z_{i+2}(E) \backslash Z_{i+1}(E)$, there exists an element $y_{0}$ in $E$ such that $\left[x, y_{0}\right] \in Z_{i+1}(E)$ and $\left[x, y_{0}\right] \notin Z_{i}(E)$. By the induction hypothesis, $\left[x, y_{0}\right] \in Z_{i+1}(N) \backslash Z_{i}(N)$.

We claim that $x \notin Z_{i+1}(N)$. Write $k$ for the index of $N$ in $E$. Then, $y_{0}^{k} \in N$. Assume $\left[x, y_{0}^{k}\right] \in Z_{i}(N)$. Remark that, as $\left[x, y_{0}\right] \in Z_{i+1}(E)$, for each $z \in E$, $\left[\left[x, y_{0}\right], z\right] \in Z_{i}(E) \subset Z_{i}(N)$. Therefore, in $N / Z_{i}(N)$, we obtain

$$
\begin{aligned}
1=\left[x, y_{0}^{k}\right] Z_{i}(N) & \stackrel{(1)}{=}\left(\prod_{j=0}^{k-1}\left[x, y_{0}\right]_{0}^{y_{0}^{j}}\right) Z_{i}(N) \\
& =\left[x, y_{0}\right]^{k} Z_{i}(N)=\left(\left[x, y_{0}\right] Z_{i}(N)\right)^{k} .
\end{aligned}
$$

As $N / Z_{i}(N)$ is torsion-free , $\left[x, y_{0}\right] \in Z_{i}(N)$ which is a contradiction. Hence, $\left[x, y_{0}^{k}\right] \notin Z_{i}(N)$ and $x \notin Z_{i+1}(N)$.

(4) If $N$ is of class $c$, then $Z_{c}(N)=N$ and consequently $Z_{c+1}(E) \backslash Z_{c}(E) \subset$ $N \backslash N=\{1\}$ or $Z_{c+1}(E)=Z_{c}(E)$. Then $E_{c+1}=E_{c}$.

For an essential extension $1 \rightarrow N \rightarrow E \rightarrow F \rightarrow 1$, defining the AC-group $E$, we now know that there exists a minimal $\ell \in \mathbb{N}$ such that $E_{\ell+i}=E_{\ell}$, for all $i \in \mathbb{N}$. Moreover, according to Lemma 3.4, $0 \leq \ell \leq c(c$ the nilpotency class of $N)$. Let us call this $\ell$ the length of the $A C$-series of $E$.

As a direct consequence of Lemma 1.2, we have

Corollary 3.5. Let $E$ be an $A C$-group and $\ell$ the length of the associated $A C$-series of $E$. If $b_{1}\left(E_{i}\right)=0$ for some $1 \leq i<c$, then $\ell \leq i$.

\section{Automorphisms of Almost CRYstallographic Groups II}

Write $L_{1}$ for the $\mathrm{Mal}^{\prime}$ cev completion of $N_{1}=N / Z(E)$, and also assume that $Z(E)$, which is torsion-free abelian, is of $\operatorname{rank} k$, i.e. $Z(E) \cong \mathbb{Z}^{k}$. We can now state

Theorem 4.1. Let $1 \rightarrow N \rightarrow E \rightarrow F \rightarrow 1$ be an essential extension and $L$ the Mal'cev completion of $N$. Then, 
(1) if $\ell=0$ and $L^{F}=\{1\}$, there exists a faithful representation $\operatorname{Aut}(E) \hookrightarrow$ $\operatorname{Aff}(L)$;

(2) if $\ell \neq 0, b_{1}\left(E_{1}\right)=0$ and $L_{1}^{F}=\{1\}$, there exists a faithful representation of $\operatorname{Aut}(E) \hookrightarrow \operatorname{Gl}(k, \mathbb{Z}) \times \operatorname{Aff}\left(L_{1}\right)$

Proof. The case $\ell=0$ ( $E$ is centerless) was treated already before.

Assume $\ell>0$. First remark that if $b_{1}\left(E_{1}\right)=0$, then, because of Corollary 3.5 , the AC-series of $E$ has length $\ell=1$ (or $Z\left(E_{1}\right)=\{1\}$ ). An automorphism $\sigma$ of $E$ restricts to an automorphism $\varphi(\sigma)$ of $Z(E)$, and consequently induces an automorphism $\bar{\sigma}$ of $E_{1}$. So, $\sigma$ gives rise to the following commutative diagram:

$$
\begin{array}{ccccccccc}
1 & \rightarrow & Z(E) & \rightarrow & E & \rightarrow & E_{1} & \rightarrow & 1 \\
& & \downarrow \varphi(\sigma) & & \downarrow & & & \downarrow & \\
& & & & & & & \\
1 & \rightarrow & Z(E) & \rightarrow & E & \rightarrow & E_{1} & \rightarrow & 1
\end{array}
$$

Since $L_{1}^{F}$ is trivial, there exists a faithful representation $\rho(\sigma)$ of $\bar{\sigma}$ in $\operatorname{Aff}\left(L_{1}\right)$. Clearly $\varphi$ and $\rho$ are homomorphisms. It remains to show that $\varphi \times \rho: \operatorname{Aut}(E) \rightarrow$ $\operatorname{Gl}\left(k_{0}, \mathbb{Z}\right) \times \operatorname{Aff}\left(L_{1}\right)$ is injective. An automorphism $\sigma \in \operatorname{Aut}(E)$ lies in the kernel of $\varphi \times \rho$ if and only if $\sigma$ induces the identity on both $Z(E)$ and $E_{1}$. The subgroup of all such automorphisms in $\operatorname{Aut}(E)$ is isomorphic to $Z^{1}\left(E_{1}, Z(E)\right)$ (e.g. see [IM94]). Since $Z(E)$ is a trivial $E_{1}$-module and $b_{1}\left(E_{1}\right)=0$ (use $(2)$ ), we conclude that this $Z^{1}\left(E_{1}, Z(E)\right)$ is trivial and, hence, that $\varphi \times \rho$ is faithful.

Remark 4.2. If $E$ is crystallographic, Theorem 4.1 reduces to the result found in [Lee82, Lemma 1].

AB-groups $E$ are precisely the fundamental groups of the infra-nilmanifolds. These manifolds are aspherical. In view of this, the study of $\operatorname{Out}(E)$ can be considered of special interest (e.g. [CR77], [IM94]). In a search to represent and to control $\operatorname{Out}(E)$, Theorem 4.1 can be most useful. This is certainly true in a situation where $E_{\ell}$ is crystallographic. A good algebraic source of examples of $A B$-groups is found in [DIKL93] (all isomorphism types in dimension 3) and in [Dek93] (dimension $\leq 4$ ). The following example uses an AB-group of type $\mathbf{2 7}$ in [Dek93].

Example 4.3. Consider the group $E$ presented as:

$$
\begin{array}{clll}
E:\langle a, b, c, d, \alpha, \beta \| & {[b, a]=d^{4}} & {[c, a]=1} & {[c, b]=1} \\
& {[d, a]=1} & {[d, b]=1} & {[d, c]=1} \\
& \alpha a=a^{-1} \alpha & \alpha b=b^{-1} \alpha & \alpha c=c \alpha \\
& \alpha d=d \alpha & \alpha^{2}=d & \\
& \beta a=a \beta & \beta b=b^{-1} \beta & \beta c=c \beta \\
& \beta d=d^{-1} \beta & \beta^{2}=c & \alpha \beta=\beta \alpha d^{-1}
\end{array}
$$

$E$ fits into an essential extension

$$
1 \rightarrow N \rightarrow E \rightarrow F \cong \mathbb{Z}_{2} \times \mathbb{Z}_{2} \rightarrow 1
$$

where $N$ is a 2-step nilpotent group of rank 4 given by

$$
N:\left\langle a, b, c, d \|[b, a]=d^{4},[c, a]=[c, b]=[d, a]=[d, b]=[d, c]=1\right\rangle .
$$

The center $Z(E)$ of $E$ is the subgroup generated by $c$, and hence:

$$
\begin{array}{clll}
E_{1}:\langle a, b, d, \alpha, \beta \| & {[b, a]=d^{4}} & {[d, a]=1} & {[d, b]=1} \\
& \alpha a=a^{-1} \alpha & \alpha b=b^{-1} \alpha & \alpha d=d \alpha \\
& \beta a=a \beta & \beta b=b^{-1} \beta & \beta d=d^{-1} \beta \\
\alpha^{2}=d & \beta^{2}=1 & \alpha \beta=\beta \alpha d^{-1}
\end{array}
$$


$E_{1}$ is a centerless, 3-dimensional AC-group (type number 6 in [DIKL93]). The Mal'cev completion of its Fitting subgroup is the Heisenberg group $H$ (see also 2.2). We leave it to the reader to verify that $b_{1}\left(E_{1}\right)=0$ and that $H^{F}=\{1\}$ (use $2.3)$.

We know that $E_{1}$ can be embedded in $\operatorname{Aff}(H)=H \rtimes \operatorname{Aut}(H)$. We leave it to the reader to verify that there is a faithful representation $\operatorname{Aff}(H) \hookrightarrow \operatorname{Aff}\left(\mathbb{R}^{3}\right)$ defined as follows: an affine transformation $(h, \alpha) \in H \rtimes \operatorname{Aut}(H)$ s.t.

$$
h=A^{x} B^{y} C^{z} \in H \text { and } \alpha: H \rightarrow H:\left\{\begin{array}{l}
A \mapsto A^{\alpha_{11}} B^{\alpha_{21}} C^{p_{1}} \\
B \mapsto A^{\alpha_{12}} B^{\alpha_{22}} C^{p_{2}}
\end{array}\right.
$$

is represented in $\operatorname{Aff}\left(\mathbb{R}^{3}\right)$ as:

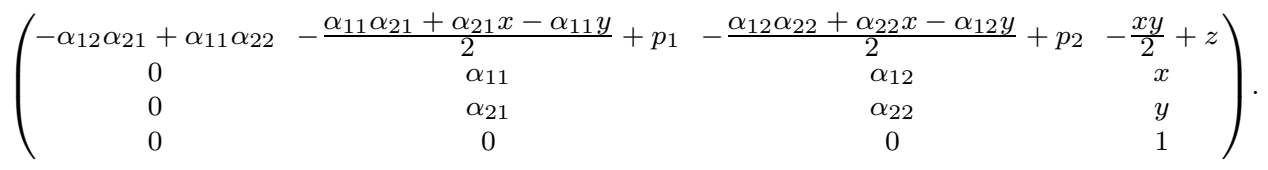

Observe that the following is a faithful representation of $E_{1}$ into $\operatorname{Aff}(H)$ (thus, realising $E_{1}$ as a genuine $\mathrm{AC}$-group):

$$
\begin{gathered}
a=\left(\begin{array}{cccc}
1 & 0 & -\frac{1}{2} & 0 \\
0 & 1 & 0 & 1 \\
0 & 0 & 1 & 0 \\
0 & 0 & 0 & 1
\end{array}\right), \quad b=\left(\begin{array}{cccc}
1 & \frac{1}{2} & 0 & 0 \\
0 & 1 & 0 & 0 \\
0 & 0 & 1 & 1 \\
0 & 0 & 0 & 1
\end{array}\right), \quad d=\left(\begin{array}{cccc}
1 & 0 & 0 & \frac{1}{4} \\
0 & 1 & 0 & 0 \\
0 & 0 & 1 & 0 \\
0 & 0 & 0 & 1
\end{array}\right), \\
\alpha=\left(\begin{array}{cccc}
1 & 0 & 0 & \frac{1}{8} \\
0 & -1 & 0 & 0 \\
0 & 0 & -1 & 0 \\
0 & 0 & 0 & 1
\end{array}\right), \beta=\left(\begin{array}{cccc}
-1 & 0 & 0 & 0 \\
0 & 1 & 0 & 0 \\
0 & 0 & -1 & 0 \\
0 & 0 & 0 & 1
\end{array}\right) .
\end{gathered}
$$

Given an automorphism $\sigma$ of $E$, we can compute, using this representation of $E_{1}$, the (unique) element of $\operatorname{Aff}(H)$ which realises, through conjugation, the induced $E_{1}$-automorphism. The image of $c$ under $\sigma$ then completes the representation of $\sigma$ into $\mathrm{Gl}(1, \mathbb{Z}) \times \operatorname{Aff}\left(\mathbb{R}^{3}\right)$.

In [IM94] we present a systematic method to study $\operatorname{Aut}(E)$ and $\operatorname{Out}(E)$ in terms of commutative diagrams. Using the information there, we obtain a short exact sequence

$$
1 \rightarrow \mathbb{Z}_{2} \rightarrow \operatorname{Out}(E) \rightarrow Q \rightarrow 1
$$

where $Q$ fits in

$$
1 \rightarrow \mathbb{Z}_{2} \times \mathbb{Z}_{2} \rightarrow Q \rightarrow \mathbb{Z}_{2} \times \mathbb{Z}_{2} \rightarrow 1
$$


In fact, $\operatorname{Out}(E)$ is generated by the images of the following $E$-automorphisms:

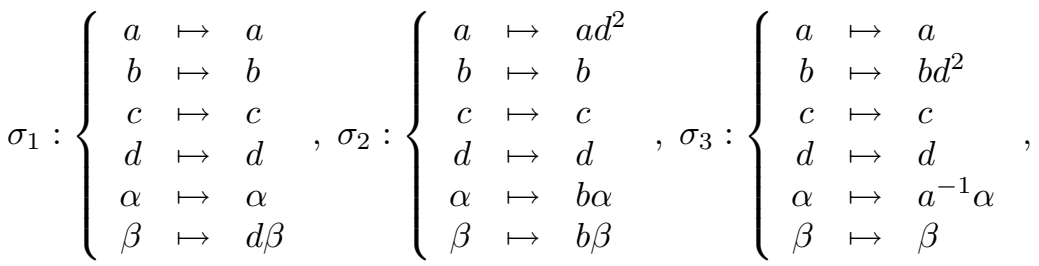

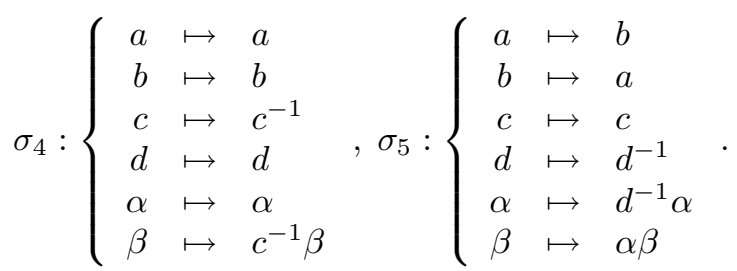

These automorphisms are represented in $\operatorname{Gl}(1, \mathbb{Z}) \times \operatorname{Aff}\left(\mathbb{R}^{3}\right)$ as

$$
\begin{gathered}
\sigma_{1}=\left(\begin{array}{ccccc}
1 & 0 & 0 & 0 & 0 \\
0 & 1 & 0 & 0 & \frac{1}{8} \\
0 & 0 & 1 & 0 & 0 \\
0 & 0 & 0 & 1 & 0 \\
0 & 0 & 0 & 0 & 1
\end{array}\right), \sigma_{2}=\left(\begin{array}{ccccc}
1 & 0 & 0 & 0 & 0 \\
0 & 1 & \frac{1}{4} & 0 & 0 \\
0 & 0 & 1 & 0 & 0 \\
0 & 0 & 0 & 1 & \frac{1}{2} \\
0 & 0 & 0 & 0 & 1
\end{array}\right), \sigma_{3}=\left(\begin{array}{ccccc}
1 & 0 & 0 & 0 & 0 \\
0 & 1 & 0 & \frac{1}{4} & 0 \\
0 & 0 & 1 & 0 & -\frac{1}{2} \\
0 & 0 & 0 & 1 & 0 \\
0 & 0 & 0 & 0 & 1
\end{array}\right), \\
\sigma_{4}=\left(\begin{array}{ccccc}
-1 & 0 & 0 & 0 & 0 \\
0 & 1 & 0 & 0 & 0 \\
0 & 0 & 1 & 0 & 0 \\
0 & 0 & 0 & 1 & 0 \\
0 & 0 & 0 & 0 & 1
\end{array}\right), \sigma_{5}=\left(\begin{array}{ccccc}
1 & 0 & 0 & 0 & 0 \\
0 & -1 & 0 & 0 & \frac{1}{16} \\
0 & 0 & 0 & 1 & 0 \\
0 & 0 & 1 & 0 & 0 \\
0 & 0 & 0 & 0 & 1
\end{array}\right) .
\end{gathered}
$$

Observe that a conjugation $\mu\left(a^{\alpha_{1}} b^{\alpha_{2}} d^{\alpha_{3}}\right)\left(\alpha_{i} \in \mathbb{Z}\right)$ in $E$ is represented as

$$
\mu\left(a^{\alpha_{1}} b^{\alpha_{2}} d^{\alpha_{3}}\right)=\left(\begin{array}{ccccc}
1 & 0 & 0 & 0 & 0 \\
0 & 1 & \frac{\alpha_{2}}{2} & -\frac{\alpha_{1}}{2} & -\frac{\alpha_{1} \alpha_{2}}{2}+\frac{\alpha_{3}}{4} \\
0 & 0 & 1 & 0 & \alpha_{1} \\
0 & 0 & 0 & 1 & \alpha_{2} \\
0 & 0 & 0 & 0 & 1
\end{array}\right) .
$$

Now it is not too hard to verify that $\sigma_{1}^{2}=\mu(d), \sigma_{2}^{2}=\mu(b), \sigma_{3}^{2}=\mu\left(a^{-1}\right), \sigma_{4}^{2}=$ $\sigma_{5}^{2}=1,\left[\sigma_{5}, \sigma_{1}\right]=\mu(d), \sigma_{5} \sigma_{2} \sigma_{5}^{-1}=\mu(a) \sigma_{3}, \sigma_{5} \sigma_{3} \sigma_{5}^{-1}=\mu\left(b^{-1}\right) \sigma_{2},\left[\sigma_{3}, \sigma_{2}\right]=\mu(d)$, $\left[\sigma_{5}, \sigma_{4}\right]=\left[\sigma_{4}, \sigma_{1}\right]=\left[\sigma_{4}, \sigma_{2}\right]=\left[\sigma_{4}, \sigma_{3}\right]=\left[\sigma_{3}, \sigma_{1}\right]=\left[\sigma_{2}, \sigma_{1}\right]=1$. From this, finally, it can be deduced that $\operatorname{Out}(E)$ is generated by the projections of $\sigma_{1}, \sigma_{4}, \sigma_{2} \sigma_{5}$ and $\sigma_{2}$ and is isomorphic to $\mathbb{Z}_{2} \times \mathbb{Z}_{2} \times \mathcal{D}_{4}\left(\mathcal{D}_{4}\right.$ is the dihedral group of order 8).

\section{REFERENCES}

[Aus60] Louis Auslander, Bieberbach's theorem on space groups and discrete uniform subgroups of Lie groups, Ann. of Math. (2) 71 (1960), 579-590. MR 22:12161

[CR77] Pierre E. Conner and Frank Raymond, Deforming homotopy equivalences to homeomorphisms in aspherical manifolds, Bull. Amer. Math. Soc. 83 (1977), 36-85. MR $\mathbf{5 7 : 7 6 2 9}$

[Dek93] Karel Dekimpe, Almost Bieberbach groups: cohomology, construction and classification, Doctoral Thesis, K.U. Leuven, 1993.

[DIKL93] Karel Dekimpe, Paul Igodt, Suhyung Kim, and Kyung Bai Lee, Affine structures for closed 3-dimensional manifolds with nil-geometry, Quart. J. Math. Oxford. Ser. (2) 46 (1995), 141-167. 
[DIM93] Karel Dekimpe, Paul Igodt, and Wim Malfait, On the fitting subgroup of almost crystallographic groups, Tijdschrift van het Belgisch Wiskundig Genootschap B (1993), 35-47. CMP 95:07

[GS92] Fritz Grunewald and Dan Segal, On affine crystallographic groups, preprint, 1992.

[HS86] Howard Hiller and Chih-Han Sah, Holonomy of flat manifolds with $b_{1}=0$, Quart. J. Math. Oxford Ser. (2) 37 (1986), 177-187. MR 88f:53073

[IM94] Paul Igodt and Wim Malfait, Extensions realising a faithful abstract kernel and their automorphisms, Manuscripta Math. 84 (1994), 135-161. CMP 94:15

[Lee82] Kyung Bai Lee, Geometric realization of $\pi_{0} \epsilon(m)$, Proc. Amer. Math. Soc. 86 (1982), 353-357. MR 84m:57026

[Lee88] _ There are only finitely many infra-nilmanifolds under each nilmanifold, Quart. J. Math. Oxford Ser. (2) 39 (1988), 61-66. MR 89f:22018

[LR85] Kyung Bai Lee and Frank Raymond, Rigidity of almost crystallographic groups, Contemp. Math. vol. 44, Amer. Math. Soc., Providence, RI, 1985, pp. 73-78. MR 87d:57026

[Mal51] Anatoliǐ I. Mal'cev, On a class of homogeneous spaces, Transl. Math. Monographs, vol. 39, Amer. Math. Soc., Providence, RI, 1951, pp. 1-33. MR 12:589e

[Pas77] Donald S. Passman, The algebraic structure of group rings, Pure and Applied Mathematics, Wiley, New York, 1977. MR 81d:16001

[Seg83] Daniel Segal, Polycyclic groups, Cambridge Univ. Press, Cambridge, 1983. MR 85h:20003

Department of Mathematics, Katholieke Universiteit Leuven Campus Kortrijk, UniVersitaire Campus, B-8500 Kortrijk, Belgium

E-mail address: paul.igodt@kulak.ac.be 